\title{
A GEM-based Time Projection Chamber for the AMADEUS experiment
}

\section{Poli Lener* Laboratori Nazionali di Frascati - INFN, Frascati, Italy}

E-mail: marco.polileneralnf.infn.it

M. Bazzi, G. Corradi, C. Curceanu, A. D’Uffizi, C. Paglia, E. Sbardella, A. Scordo, D. Tagnani

Laboratori Nazionali di Frascati - INFN, Frascati, Italy

\section{A. Romero Vidal,}

Laboratori Nazionali di Frascati - INFN, Frascati, Italy

Universidad de Santiago de Compostela, Santiago de Compostela, Spain

\section{J. Zmeskal}

Stefan Meyer Institut fur sübatomare Physik, Vienna, Austria

In this paper we present the R\&D activity on a new GEM-based TPC prototype for AMADEUS, a new experimental proposal at the DA $\Phi N E \Phi$-factory at the Laboratori Nazionali di Frascati (INFN), aiming to perform measurements of the low-energy negative kaons interactions in nuclei. Such innovative detector will equip the inner part of the experiment in order to perfom a better reconstruction of the primary vertex and the secondary particles tracking.

A $10 \times 10 \mathrm{~cm}^{2}$ prototype with a drift gap up to $15 \mathrm{~cm}$ was realized and succesfully tested at the $\pi \mathrm{M} 1$ beam facility of the Paul Scherrer Institut (PSI) with low momentum hadrons. The measurements of the detector efficiency and spatial resolution have been performed. The results as a function of the gas gain, drift field, front-end electronic threshold and particle momentum are reported and discussed.

International Winter Meeting on Nuclear Physics,

21-25 January 2013

Bormio, Italy

\footnotetext{
*Speaker.
} 


\section{Introduction}

An important, yet unsolved problem, in hadron physics is how the hadron masses and interactions change in the nuclear medium. This topic could be investigated by means of "inmedium hadron-mass spectroscopy", producing bound states of a hadron by which to deduce the hadron-nucleus potential and the in-medium hadron masses. The AMADEUS (Antikaon Matter At DAФNE Experiments with Unraveling Spectroscopy) experiment [1],[2] will study the low energy interactions of kaons with nucleons and nuclei. The AMADEUS setup will be implemented inside the KLOE [3] Drift Chamber (DC), in the free space between the beam pipe and the DC entrance wall. Three main components of the experimental setup are under development: a high density cryogenic gaseous target, a trigger system [4], and an inner tracker, namely a Time Projection Chamber equipped with Gas Electron Multiplier [5], to be positioned inside the KLOE DC, which will perform a better reconstruction of the primary vertex and the secondary particles tracking. A representation of the dedicated AMADEUS setup surrounding the beam pipe within KLOE detector is given in Figure 1.
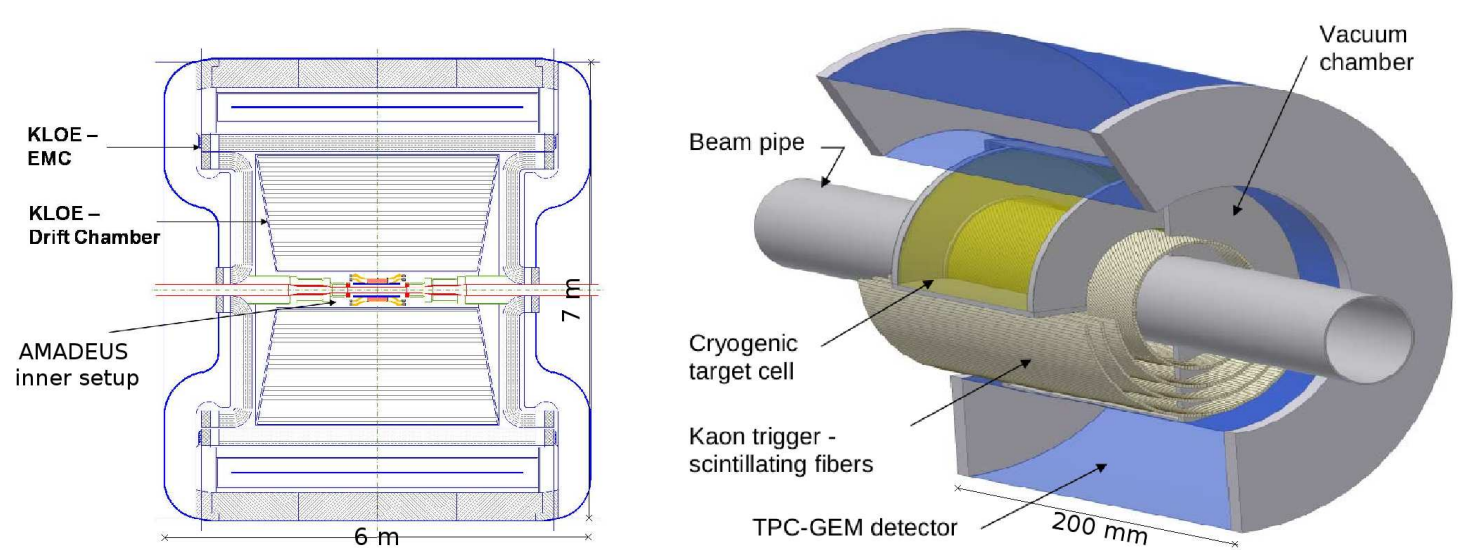

Figure 1: Cross-section of the KLOE detector including the AMADEUS inner setup inside the Drift Chamber (left) and the inner setup of AMADEUS. From the beam pipe to the outer region: the Kaonic Trigger, the Gas Target and the GEM based-TPC.

The GEM-based TPC (TPG) will be $20 \mathrm{~cm}$ long with an inner diameter of $8 \mathrm{~cm}$ and an outer one of $40 \mathrm{~cm}$. The main requirements for the TPG can be summarized as:

- a spatial resolution better than $200 \mu \mathrm{m}$ in $\mathrm{X}-\mathrm{Y}$ and $300 \mu \mathrm{m}$ in Z;

- a detector material budget lower than $0.5 \%$ of $\mathrm{X}_{0}$;

- a rate capability of $\sim 5 \mathrm{kHz} / \mathrm{cm}^{2}[6]$;

Since most of the above requirements are easily fulfilled by a TPG, the R\&D activity at the Laboratori Nazionali di Frascati (INFN) is mainly focused on the choice of the gas mixture, in order to achieve the highest spatial resolution with a $0.5 \mathrm{~T}$ value of magnetic field, and on the design of the detector readout. 
A TPG prototype of $10 \times 10 \mathrm{~cm}^{2}$ with a drift gap up to $15 \mathrm{~cm}$ was realized and tested both in laboratory and at Paul Scherrer Institut (PSI). Detail of the prototype construction can be found in Ref. [7]. The experimental setup of the test beam and the choice of the gas mixtures are described in Sec. 2. In Sec. 3 the detector performances obtained at the $\pi \mathrm{M} 1$ beam facility, in terms of detector efficiency and spatial resolution, are presented and discussed. The paper ends with conclusions.

\section{Experimental setup}

The performance of the TPG prototype was studied at the $\pi \mathrm{M} 1$ beam facility of the PSI without magnetic field. The $\pi \mathrm{M} 1$ beam is a quasi-continuous high-intensity secondary beam providing hadrons with a momentum resolution of $\sim 1 \%$.

The measurement of the detector efficiency and spatial resolution was performed with a beam rate of $\sim 200 \mathrm{~Hz}$.

The prototype readout is composed by 4 rows of 32 pads of $\sim 3 \times 3 \mathrm{~mm}^{2}$ each. Each pad was connected to a front-end board based on CARIOCA-GEM chip [8]. The discriminator threshold on the front-end electronics was set to $\sim 3.5 \mathrm{fC}$ and $\sim 5 \mathrm{fC}$.

During this test beam isobutane-based gas mixtures were used. As shown in Tab. 1, such gas mixtures are charactezed by:

- a large primary ionisation;

- a high drift velocity;

- a high Townsend coefficient;

- a moderate longitudinal and transversal diffusions;

- a very low attachment coefficient;

\begin{tabular}{|c|c|c|c|c|c|c|c|}
\hline $\begin{array}{c}\text { Gas } \\
\text { Mixture }\end{array}$ & $\begin{array}{c}\text { Average } \\
\text { Townsend Coeff. } \\
{[1 / \mathrm{V}]}\end{array}$ & $\begin{array}{c}\text { Drift Velocity } \\
\text { at } 200 \mathrm{~V} / \mathrm{cm} \\
{[\mu \mathrm{m} / \mathrm{ns}]}\end{array}$ & $\begin{array}{r}\text { Longitudinal } \& \\
\text { at } 20 \\
{[\mu \mathrm{m}}\end{array}$ & $\begin{array}{l}\text { ransversal Diff. } \\
\mathrm{V} / \mathrm{cm} \\
{[\sqrt{\mathrm{cm}}]}\end{array}$ & $\begin{array}{c}170 \mathrm{MeV} / \mathrm{c} \\
\text { Pion }\end{array}$ & $\begin{array}{c}\text { Cluster/cm } \\
440 \mathrm{MeV} / \mathrm{c} \\
\text { Proton }\end{array}$ & MIPs \\
\hline $\mathrm{Ar} / \mathrm{C}_{4} \mathrm{H}_{10}=80 / 20$ & $(25.2 \pm 0.2) \times 10^{-3}$ & $29 \pm 2$ & $217 \pm 16$ & $274 \pm 12$ & $45.2 \pm 2.1$ & $96.6 \pm 3.5$ & $40.0 \pm 2.0$ \\
\hline $\mathrm{Ar} / \mathrm{C}_{4} \mathrm{H}_{10}=90 / 10$ & $(\mathbf{2 7 . 2} \pm \mathbf{0 . 3}) \times 10^{-3}$ & $39 \pm 2$ & $282 \pm 7$ & $359 \pm 18$ & $37.2 \pm 1.9$ & $79.6 \pm 2.8$ & $32.8 \pm 1.8$ \\
\hline $\mathrm{Ar} / \mathrm{CO}_{2}=70 / 30$ & $(22.0 \pm 0.3) \times 10^{-3}$ & $4.51 \pm 0.02$ & $178 \pm 11$ & $175 \pm 6$ & $32.2 \pm 1.8$ & $68.8 \pm 2.6$ & $28.4 \pm 1.6$ \\
\hline
\end{tabular}

Table 1: Average of Townsend coefficient, drift velocity, diffusion coefficients and primary ionization. The values in bold were measured experimentally [7], while the normal ones were obtained by Garfield simulation [9]. The Ar/ $\mathrm{CO}_{2}=70 / 30$ gas mixture and MIPs are reported for comparison.

\section{Detector Performances}

\subsection{Efficiency}

The single pad row detector efficiency was evaluated considering the fraction of the hits in a single pad row with respect to a selected track. 

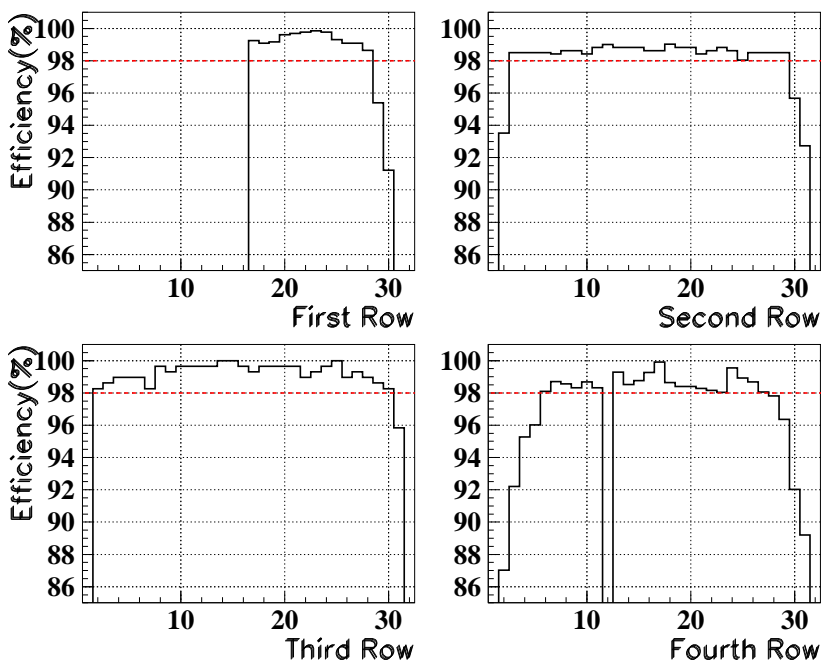

Figure 2: Single pad efficiency. Each pad is $\sim 3 \times 3 \mathrm{~mm}^{2}$.

In Fig. 2 is shown the single pad efficiency for each row. It is worth noticing that most of the pads have an efficiency larger than $98 \%$ except for the first 16 channels of the first row and one channel of the fourth row that were dead. A low and/or not full efficiency in the first and last pads of each rows and a parabolic behaviour are clearly visible. These effects were discussed in detail in Ref. [7].

The detector efficiency for the $\mathrm{Ar} / \mathrm{C}_{4} \mathrm{H}_{10}=80 / 20$ and $\mathrm{Ar} / \mathrm{C}_{4} \mathrm{H}_{10}=90 / 10$ gas mixtures as a function of the gas gain with $150 \mathrm{~V} / \mathrm{cm}$ drift field and $170 \mathrm{MeV} / \mathrm{c}$ pion beam is shown in Fig. 3. As expected, the use of $3.5 \mathrm{fC}$ front-end electronics threshold allows to reach a full efficiency at lower values of gain with respect to the higher threshold measurements.

The increase of the drift field from $150 \mathrm{~V} / \mathrm{cm}$ to $210 \mathrm{~V} / \mathrm{cm}$ with a fixed gain of $\sim 8 \times 10^{3}$ allows to increase the detector efficiency from $\sim 70 \%$ to $\sim 90 \%$ for both gas mixtures at $5 \mathrm{fC}$ threshold and from $\sim 90 \%$ to a full efficiency for $\mathrm{Ar} / \mathrm{C}_{4} \mathrm{H}_{10}=90 / 10$ gas mixture at $3.5 \mathrm{fC}$ threshold, as shown in Fig. 4. This effect can be explained by a greater collection efficiency of the primary electrons in the first GEM: indeed, when the drift field increases in the above mentioned range the diffusion of the primary electrons decreases allowing to reach the GEM holes and to give rise to the avalanche process.

The different level of efficiency between the two curves at $5 \mathrm{fC}$ threshold is due to the higher number of primary electrons produced in the drift gap in the $\mathrm{Ar} / \mathrm{C}_{4} \mathrm{H}_{10}=80 / 20$ gas mixture with respect to the $\mathrm{Ar} / \mathrm{C}_{4} \mathrm{H}_{10}=90 / 10$ one (see Tab. 1).

The efficiency for $440 \mathrm{MeV} / \mathrm{c}$ proton and $170 \mathrm{MeV} / \mathrm{c}$ pion beams for $\mathrm{Ar} / \mathrm{C}_{4} \mathrm{H}_{10}=90 / 10$ gas mixture is shown in Fig. 5. As expected, protons allow to reach an efficiency plateau at lower gas gain with respect to pions due to a higher primary ionisation. In addition, the detector efficiency with a proton beam does not seem affected by the electronic threshold. This is due to a high primary ionisation of protons which allows to produce a signal above the used discriminator thresholds.

On the contrary, the low threshold value with a pions beam allows to increase the detector efficiency 


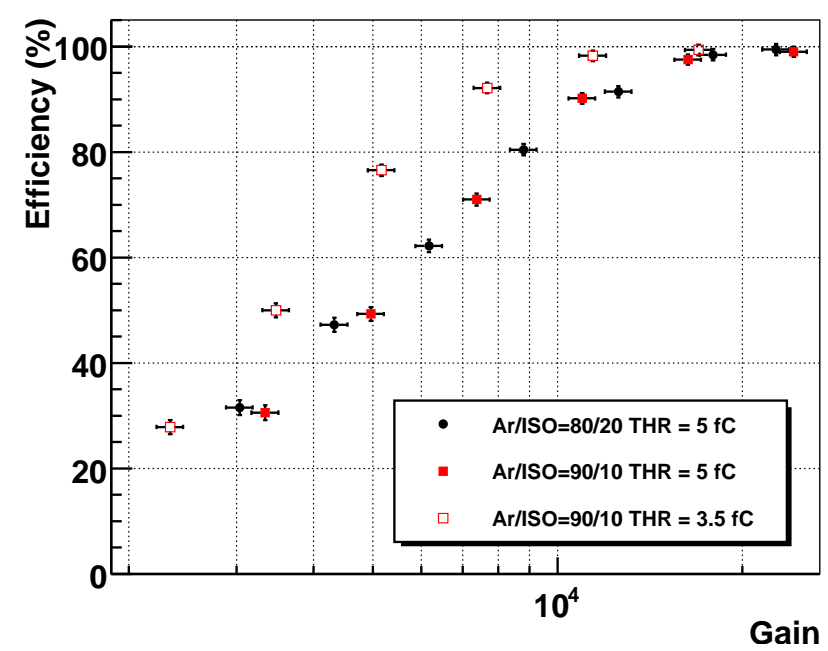

Figure 3: Detector Efficiency for the $\mathrm{Ar} / \mathrm{C}_{4} \mathrm{H}_{10}=80 / 20$ and $\mathrm{Ar} / \mathrm{C}_{4} \mathrm{H}_{10}=90 / 10$ gas mixtures as a function of the gas gain with a fixed drift field of $150 \mathrm{~V} / \mathrm{cm}$ and for a $170 \mathrm{MeV} / \mathrm{c}$ pion beam.

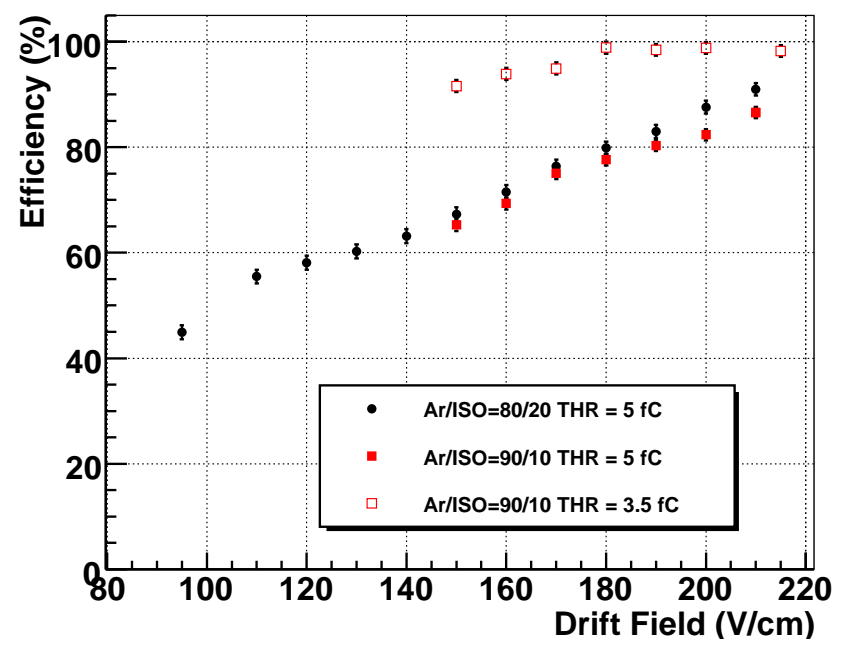

Figure 4: Detector Efficiency for the $\mathrm{Ar} / \mathrm{C}_{4} \mathrm{H}_{10}=80 / 20$ and $\mathrm{Ar} / \mathrm{C}_{4} \mathrm{H}_{10}=90 / 10$ gas mixtures as a function of the drift field with a fixed gain of $\sim 8 \times 10^{3}$ and for a $170 \mathrm{MeV} / \mathrm{c}$ pion beam.

which reaches an efficiency plateau at lower gas gain with respect to the $5 \mathrm{fC}$ measurement.

\subsection{Spatial Resolution}

The spatial resolution in the drift direction was evaluated by the residuals between the hit position in a single pad row and a selected track.

Fig. 6 shows the spatial resolution in the drift direction for $170 \mathrm{MeV} / \mathrm{c}$ pions as a function of the drift field. Since the diffusion decreases by increasing the drift field, i.e a greater collection efficiency of primary electrons into the first GEM holes, a better spatial resolution is achieved for a high drift field in the range between 100 and $210 \mathrm{~V} / \mathrm{cm}$ and a low value of the threshold. 


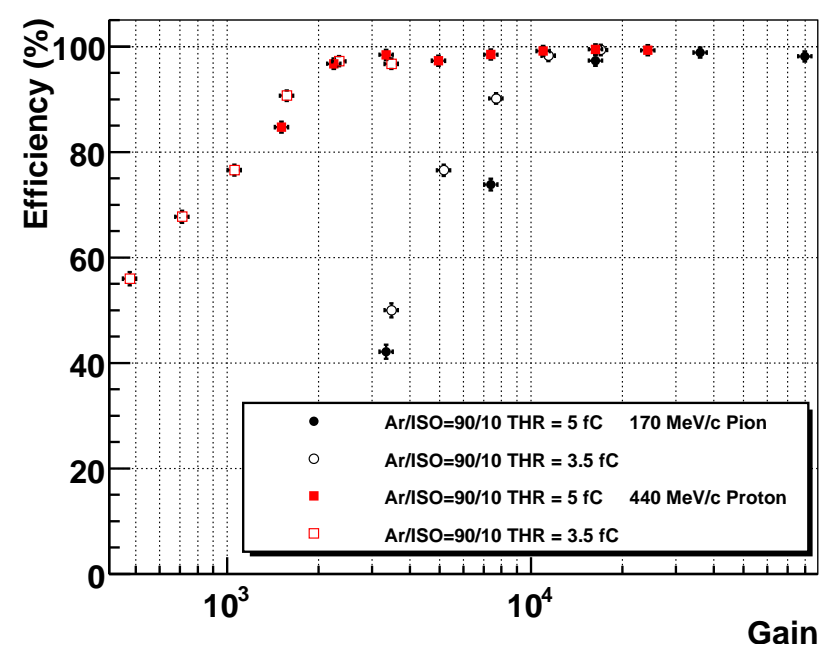

Figure 5: Detector Efficiency for protons of $440 \mathrm{MeV} / \mathrm{c}$ and pions of $170 \mathrm{MeV} / \mathrm{c}$ as a function of the gas gain. The gas mixture is $\mathrm{Ar} / \mathrm{C}_{4} \mathrm{H}_{10}=90 / 10$ and the drift field is set to $150 \mathrm{~V} / \mathrm{cm}$.

Due to a lower diffusion of the $\mathrm{Ar} / \mathrm{C}_{4} \mathrm{H}_{10}=80 / 20$ gas mixture with respect to $\mathrm{Ar} / \mathrm{C}_{4} \mathrm{H}_{10}=90 / 10$ one, a better spatial resolution is reached at $5 \mathrm{fC}$ of threshold.

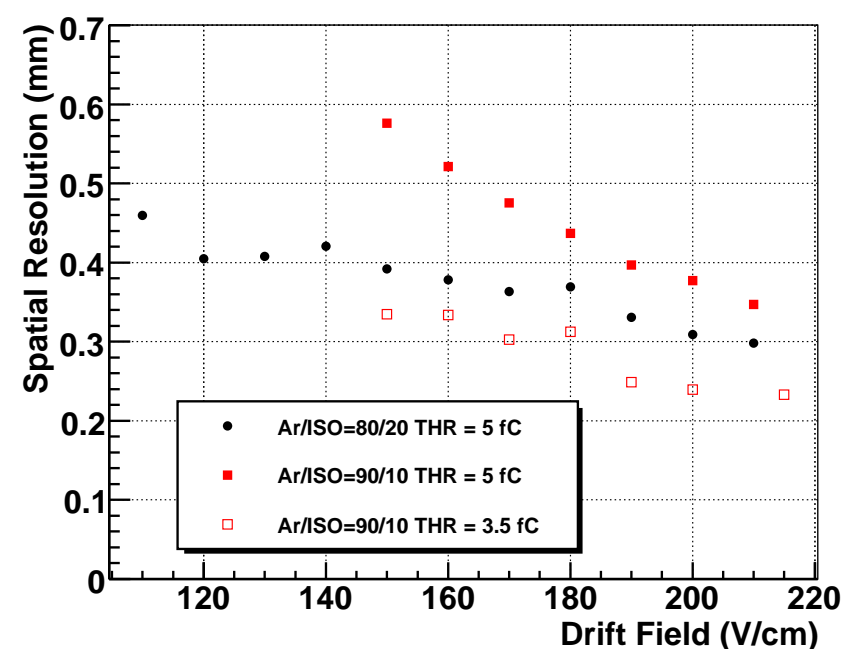

Figure 6: Spatial resolution for the $\mathrm{Ar} / \mathrm{C}_{4} \mathrm{H}_{10}=80 / 20$ and $\mathrm{Ar} / \mathrm{C}_{4} \mathrm{H}_{10}=90 / 10$ gas mixtures as a function of the drift field with a fixed gain of $\sim 8 \times 10^{3}$ and for a $170 \mathrm{MeV} / \mathrm{c}$ pion beam.

The spatial resolution for $170 \mathrm{MeV} / \mathrm{c}$ pions as a function of the gas gain at fixed drift field is shown in Fig. 7. For high threshold and for both gas mixtures the spatial resolution seems to be not affected by the detector gain, while this does not occur with a lower threshold value. A possible explanation is that the collection efficiency of primary ionisation into the first GEM holes increases as the detector gain increases until the signal is above the discrimination threshold. When this happens a better spatial resolution is achieved while it reaches a plateau when the signal is comparable 
with the electronic threshold.

Such explanation is confirmed by the $\mathrm{Ar} / \mathrm{C}_{4} \mathrm{H}_{10}=90 / 10$ gas mixture measurements, in which the spatial resolution reaches the same level at very low gain $\left(<3 \times 10^{3}\right)$ and regardless of the used threshold.

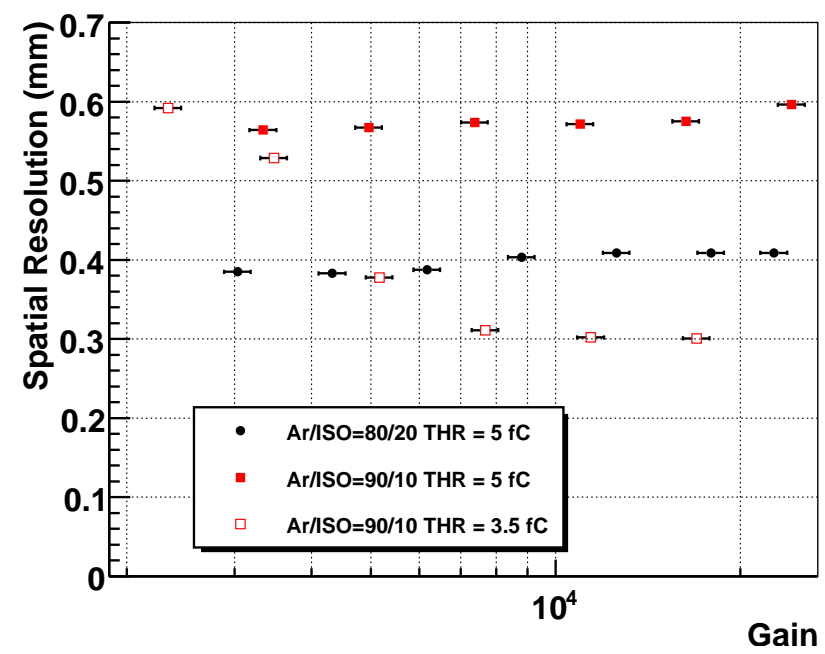

Figure 7: Spatial resolution for the $\mathrm{Ar} / \mathrm{C}_{4} \mathrm{H}_{10}=80 / 20$ and $\mathrm{Ar} / \mathrm{C}_{4} \mathrm{H}_{10}=90 / 10$ gas mixtures as a function of gas gain with a fixed drift field of $150 \mathrm{~V} / \mathrm{cm}$ and $170 \mathrm{MeV} / \mathrm{c}$ pions.

The effect of the ionazing particle on the spatial resolution was evaluated comparing 440 $\mathrm{MeV} / \mathrm{c}$ proton beam with $170 \mathrm{MeV} / \mathrm{c}$ pion one, as shown in Fig. 8. As explained above, the spatial resolution seems not so sensible to a gain change with $5 \mathrm{fC}$ threshold value, regardless of the ionazing particle. Moreover, the larger ionization produced by protons with respect to pions allows to reach a better spatial resolution of about a factor 2 .

With a $3.5 \mathrm{fC}$ of threshold, the spatial resolution for pions reaches at high gain the same level obtained with protons. This effect suggest that the spatial resolution reaches a limit value for the $\mathrm{Ar} / \mathrm{C}_{4} \mathrm{H}_{10}=90 / 10$ gas mixture.

\section{Conclusion}

The R\&D activity on a GEM-based TPC for the inner region of the AMADEUS setup has started at the Laboratori Nazionali di Frascati (INFN). A TPG prototype with a drift gap up to 15 $\mathrm{cm}$ has been successfully produced and tested at the $\pi \mathrm{M} 1$ beam facility of the Paul Scherrer Institut with low momentum pion and proton beams.

The measurement of the detector performances, in terms of efficiency and spatial resolution as a function of the gas gain, drift field, front-end electronics threshold and particle momentum, has been reported and discussed in detail. A detection efficiency of $99 \%$ and a spatial resolution of 240 $\mu \mathrm{m}$ have been achieved, compatible with the foreseen values for AMADEUS. 


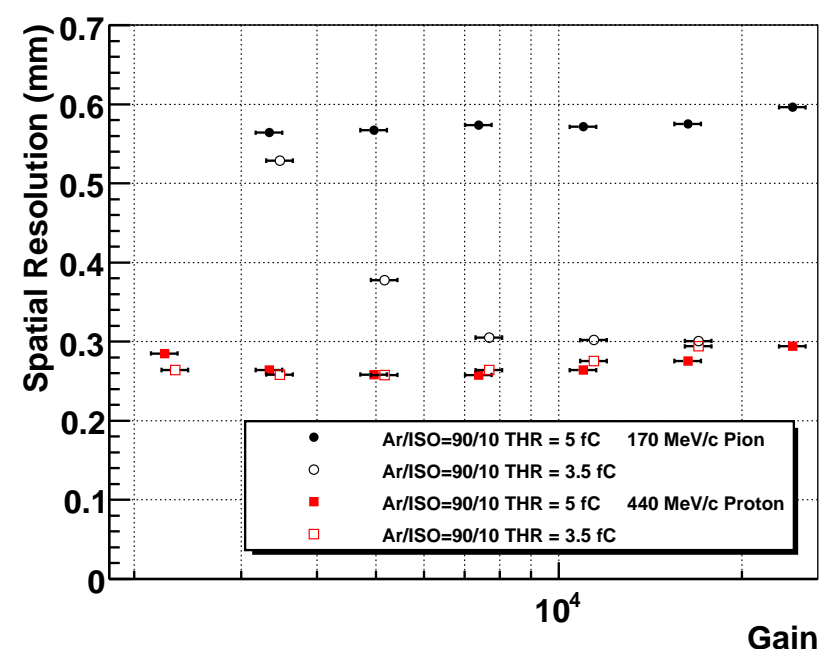

Figure 8: Spatial resolution for proton of $440 \mathrm{MeV} / \mathrm{c}$ and pion of $170 \mathrm{MeV} / \mathrm{c}$ as a function of the gas gain. The gas mixture is $\mathrm{Ar} / \mathrm{C}_{4} \mathrm{H}_{10}=90 / 10$ and the drift field is set to $150 \mathrm{~V} / \mathrm{cm}$.

\section{References}

[1] AMADEUS Collaboration, AMADEUS Letter of Intent, http://www.Inf.infn.it/Infadmin/direzione/roadmap/LOI_MARCH_AMADEUS.pdf.

[2] AMADEUS Collaboration, AMADEUS Phase-1: Setup and Roll-in Proposal, LNF-07/24(IR) 2007.

[3] D. Adinolfi et al., Nucl. Instr. and Meth. A 461 (2001) 25.

[4] M. Bazzi et al., Nucl. Instr. and Meth. A 671 (2012) 125.

[5] F. Sauli, Nucl. Instr. and Meth. A 386 (1997) 531.

[6] M. Poli Lener, Triple-GEM detectors for the innermost region of the muon apparatus at the LHCb experiment, Ph.D Thesis, CERN-THESIS-2006-13.

[7] M. Poli Lener et al., Performances of a GEM-based Time Projection Chamber prototype for the AMADEUS experiment, arXiv:1302.3054 [physics.ins-det].

[8] A. Alfonsi et al., IEEE NSS MIC Vol. 6 (2007) 4671.

[9] R. Veenhof, Nucl. Instr. and Meth. A 419 (1998) 726. 